\title{
Motion Integration for Ocular Pursuit Does Not Hinder Perceptual Segregation of Moving Objects
}

\author{
Zhenlan Jin, ${ }^{1}$ Scott N.J. Watamaniuk, ${ }^{2}$ Aarlenne Z. Khan, ${ }^{3}$ Elena Potapchuk, ${ }^{4}$ and Stephen J. Heinen ${ }^{4}$ \\ ${ }^{1}$ University of Electronic Science and Technology of China, Sichuan, China 610051, ${ }^{2}$ Wright State University, Dayton, Ohio 45435, ${ }^{3}$ Queen's University, \\ Ontario, Canada K7L 3N6, and ${ }^{4}$ The Smith-Kettlewell Eye Research Institute, San Francisco, California 94115
}

\begin{abstract}
When confronted with a complex moving stimulus, the brain can integrate local element velocities to obtain a single motion signal, or segregate the elements to maintain awareness of their identities. The integrated motion signal can drive smooth-pursuit eye movements (Heinen and Watamaniuk, 1998), whereas the segregated signal guides attentive tracking of individual elements in multiple-object tracking tasks (MOT; Pylyshyn and Storm, 1988). It is evident that these processes can occur simultaneously, because we can effortlessly pursue ambulating creatures while inspecting disjoint moving features, such as arms and legs, but the underlying mechanism is unknown. Here, we provide evidence that separate neural circuits perform the mathematically opposed operations of integration and segregation, by demonstrating with a dual-task paradigm that the two processes do not share attentional resources. Human observers attentively tracked a subset of target elements composing a small MOT stimulus, while pursuing it ocularly as it translated across a computer display. Integration of the multidot stimulus yielded optimal pursuit. Importantly, performing MOT while pursuing the stimulus did not degrade performance on either task compared with when each was performed alone, indicating that they did not share attention. A control experiment showed that pursuit was not driven by integration of only the nontargets, leaving the MOT targets free for segregation. Nor was a predictive strategy used to pursue the stimulus, because sudden changes in its global velocity were accurately followed. The results suggest that separate neural mechanisms can simultaneously segregate and integrate the same motion signals.
\end{abstract}

Key words: eye movements; MOT; multiobject tracking

\section{Introduction}

Smooth-pursuit eye movements follow moving objects. Smooth pursuit is traditionally studied with a small, spot stimulus to minimize confounding factors introduced into the neural signal by complicated visual stimuli, thereby allowing pure assessment of the pursuit motor substrate. However, natural pursuit objects, such as a proximal person, are usually much larger than the laboratory spot, and have limbs or other local elements that move at different velocities than the global object. Work has shown that global stimulus motion can be pursued, regardless of individual element motion (Steinbach, 1976; Watamaniuk and Heinen, 1999). Natural objects also have features that require or attract attention. These qualitative differences between natural stimuli and the spot suggest that the mechanism driving spot pursuit may be different from the mechanism driving pursuit of larger objects.

The human motion perception system integrates disparate local element velocities of moving objects to determine their

\footnotetext{
Received Nov. 19, 2013; revised March 11, 2014; accepted March 15, 2014.

Author contributions: Z.J., S.N.J.W., A.Z.K., and S.J.H. designed research; Z.J., S.N.J.W., and E.P. performed research; Z.J., S.N.J.W., A.Z.K., E.P., and S.J.H. contributed unpublished reagents/analytic tools; Z.J., S.N.J.W., and E.P. analyzed data; Z.J., S.N.J.W., A.Z.K., E.P., and S.J.H. wrote the paper.

This work was supported by NIH Grant EY021286.

The authors declare no competing financial interests.

Correspondence should be addressed to Dr Stephen J. Heinen, Smith-Kettlewell Eye Research Institute, 2318

Fillmore Street, San Francisco, CA 94115. E-mail: heinen@ski.org.

DOI:10.1523/JNEUROSCI.4867-13.2014

Copyright $\odot 2014$ the authors $\quad 0270-6474 / 14 / 345835-07 \$ 15.00 / 0$
}

global velocity. Evidence for this is that random dot cinematograms (RDCs) composed of spatially distributed dots that move with different directions and speeds, produce a motion percept equal to calculated global RDC velocity (Williams and Sekuler, 1984; Watamaniuk and Duchon, 1992). An integrated motion signal also drives smooth pursuit of RDCs, and pursuit dynamics improve with increasing motion coherence (Watamaniuk and Heinen, 1999; Stone et al., 2000; Schütz et al., 2009), dot density, and RDC area (Heinen and Watamaniuk, 1998). Furthermore, the pursuit system responds better to RDCs than to single spots, because coherent motion RDCs produce higher open-loop gain and fewer saccades than the spot (Heinen and Watamaniuk, 1998; Watamaniuk and Heinen, 1999).

Paradoxically, the brain can also attentively segregate multiple moving elements, as has been demonstrated during multiple-object tracking (MOT; Pylyshyn and Storm, 1988), which activates motion and attention structures in the brain (Howe et al., 2009). In MOT, observers attentively follow a subset of identical, independently moving stimulus elements. Approximately four elements can be accurately tracked (Pylyshyn and Annan, 2006), likely with covert attention (Pylyshyn and Storm, 1988; Yantis, 1992; Cavanagh and Alvarez, 2005; Alvarez and Franconeri, 2007; Howe et al., 2010). The smooth-pursuit system also uses attention to follow small objects that engage the fovea (Brezinová and Kendell, 1977; Acker and Toone, 1978; Khurana and Kowler, 1987; Kerzel et al., 2008). However, pursuing objects that extend into peripheral retina, such as large RDCs (Heinen and Watamaniuk, 1998; Watamaniuk and 
Heinen, 1999), requires less attention (Heinen et al., 2011; Jin et al., 2013).

Here we use a dual-task paradigm to investigate whether neural segregation and integration can occur simultaneously. Segregation was needed to attentively track a subset of elements in a small MOT stimulus, whereas integration was needed to pursue the MOT stimulus as it translated across the display. Simultaneous execution of these tasks did not degrade either pursuit or MOT performance, even when MOT difficulty taxed baseline performance, evidence that attention resources, and hence neural circuitry driving integration and segregation for these tasks, were independent. The results show that simultaneous segregation and integration occur, and suggest that different mechanisms subserve the two processes.

\section{Materials and Methods}

Subjects. Four healthy observers (one male and three females) participated in the current study, with voluntary informed consent. All had normal or corrected to normal vision and were 24-50 years old. Three of the observers were naive to the purpose of the study.

Apparatus. Visual stimuli were generated by functions from PsychToolbox (Brainard, 1997; Pelli, 1997) in MATLAB (MathWorks) on a Macintosh G4 computer and presented on a 17 inch high-resolution Nanao color monitor $(1.76 \mathrm{~min} \mathrm{arc} / \mathrm{pixel})$ at a rate of $60 \mathrm{~Hz}$. Horizontal and vertical eye position were sampled at $1000 \mathrm{~Hz}$ by an EyeLink 1000 video-based eye tracker (SR Research). The EyeLink was calibrated and validated using the standard ninepoint method included with the system. Observers used chin and forehead rests to stabilize the head and maintain a constant viewing distance of $48 \mathrm{~cm}$.

Stimuli. Stimuli consisted of 4,8 , or 10 gray dots $\left(0.2^{\circ}\right.$ diameter, luminance $\left.2.63 \mathrm{~cd} / \mathrm{m}^{2}\right)$ presented on a dark background (luminance $0.3 \mathrm{~cd} / \mathrm{m}^{2}$ ). The dots moved randomly at speeds between $3^{\circ}$ and $10^{\circ}$ s within a $9 \times 9^{\circ}$ square virtual container. Direction and speed were generated independently for each dot, and changed randomly every $300-800 \mathrm{~ms}$. Additionally, the proximity of the dots to each other was limited to not $<0.6^{\circ}$ to ensure that there were no collisions or occlusions, thereby allowing the individual dots to retain their identities. If a dot's motion in a frame would make it travel beyond the edge of the virtual container, its speed and direction were randomly reassigned so that it remained within the confines of the virtual container. For the baseline pursuit experiment, $50 \%$ of the trials (run in separate blocks) presented a gray cross $\left(0.2^{\circ}\right.$ wide, luminance $2.63 \mathrm{~cd} / \mathrm{m}^{2}$ ) at the center of the $9 \times 9^{\circ}$ region. The entire stimulus either translated across the screen at a constant speed of $7 \%$, or remained at the screen center throughout the trial.

Procedure. Trials were blocked by number of dots, whether the central cross was present or absent, and whether the entire display was moving or static. Each block comprised 60 trials and each observer completed two blocks of each condition. At the beginning of each trial, the dots appeared at their initial positions for $1 \mathrm{~s}$. Next, they started moving within the virtual container, which either remained at the center, or translated across the screen. The cross, when present, remained at the center of the container throughout the trial, and when absent, was turned off after the fixation period.

In the attentive-tracking condition (Fig. 1), half of the dots were randomly selected as MOT targets. At the start of each trial the targets were cued by color (green, luminance $2.63 \mathrm{~cd} / \mathrm{m}^{2}$ ). After $500 \mathrm{~ms}$ of motion, the targets changed to the color of the distractor dots. The stimulus continued to move for another $2.5 \mathrm{~s}$, during which time subjects were required to attentively track the MOT targets. A probe dot changed to green for the last $300 \mathrm{~ms}$ of each trial, and subjects had to identify with a key press ( 4 for "yes" and 6 for "no") whether the probe was one of the original MOT targets. The probe was equally likely to be a target or a distractor. In the control condition, there were no targets, no dots were cued, and no identification was necessary. To initiate the next trial, subjects pressed the Enter key, so they controlled the pace of the experiment.

Eye movement data analysis. Horizontal and vertical eye velocity were calculated offline from the recorded position signals by differentiating and filtering the raw eye position data (2-pole Butterworth noncausal 
filter, cutoff $=50 \mathrm{~Hz}$ ). Saccades were detected offline when eye velocity exceeded $50 \%$ s. Each saccade was removed from the velocity trace and replaced with a line that interpolated eye velocity before and after the saccade.

For the pursuit data analysis for Experiments 1 and 2, steady-state pursuit gain was computed by dividing average eye velocity $500-2700 \mathrm{~ms}$ after stimulus motion onset by stimulus velocity. This period coincides with when observers were actively tracking the MOT targets, after the cuing period but before the appearance of the probe. All statistical tests used an $\alpha$ level of 0.05 for both the eye movements and the psychophysics.

\section{Results}

We first asked whether observers could pursue a translating MOT stimulus, given it is an unconventional pursuit target. Although previously it has been shown that noisy RDCs can be pursued, in that research, all dots moved at the same speed and always had a motion vector in the global motion direction (Watamaniuk and Heinen, 1999). In this study, individual elements move at different, randomly changing velocities, and therefore their directions do not always include the global motion vector. To establish baseline pursuit of a stimulus requiring integration, we used a 10-dot MOT cloud. A small central cross that served as an explicit pursuit target was either present or absent within a block. Observers were instructed to pursue the dot cloud. No MOT task was performed and the dots were a gray color for the duration of the trial.

Consistent with previous work, observers matched the global velocity of the translating stimulus without the need for a constant velocity target. Figure $2 a$ shows representative average eye traces from one subject for two blocks of trials, one with and one without the central cross. Steady-state pursuit velocity is the same with and without the embedded pursuit target, and matches the stimulus velocity well. Figure $2 b$ summarizes mean steady-state pursuit gain for all observers in the two conditions. A paired $t$ test showed no difference between conditions $\left(t_{(7)}=0.5285, p=0.61\right)$. Therefore, the pursuit system appears capable of following the integrated global velocity of these stimuli.

Given the pursuit system can follow the integrated motion of an MOT stimulus, we were poised to ask whether the integration could occur simultaneously with segregation needed for MOT. To this end, we used a dual-task paradigm. Dual task paradigms are typically used to determine whether two processes use the same attentional resources (Navon and Gopher, 1979), and thereby shed light on whether the mechanisms underlying them are independent. It follows then that if neural integration and segregation are independent processes, it should be possible to pursue the MOT stimuli while performing the MOT task, with no decrement to the pursuit eye movement or MOT accuracy. We addressed this by having observers perform MOT while pursuing the task stimulus as it translated across the screen. Note that if there were sufficient attention for both MOT and pursuit, no deficit would be found, rendering the result inconclusive. To avoid this problem, we increased the difficulty of the MOT task to tax attentional resources by adding MOT targets and distractors. This manipulation was effective because MOT errors for the nontranslating stimulus increased as the number of elements increased.

Observers attentionally tracked 2, 4, or 5 targets in clouds of 4, 8 , or 10 dots, respectively. The number of targets was chosen to keep the probability of guessing the probe's identity constant at $50 \%$. Baseline MOT performance was established when the stimulus cloud remained centered on the screen, with no translational motion imposed. It was then compared with MOT performance

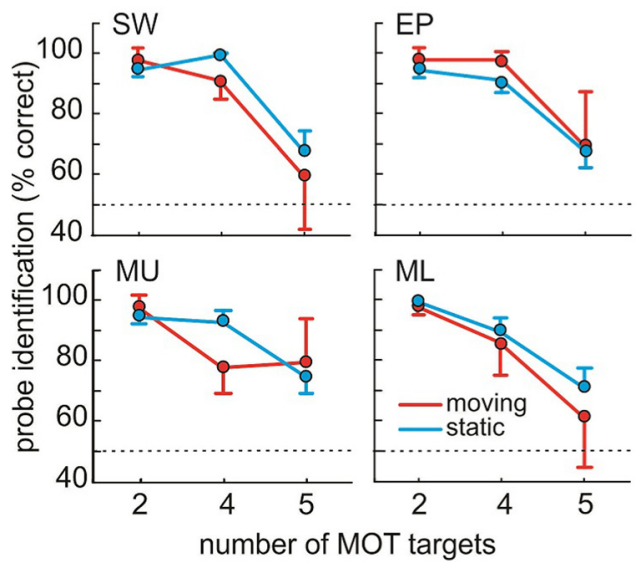

Figure 3. MOT performance as a function of number of MOT targets for both static and moving stimuli. Error bars indicate SEM. Although all observers show a decline in MOT performance as the number of tracked targets increases, there is no consistent performance decrement from pursuing the stimulus cloud. Horizontal dashed lines indicate $50 \%$ (chance) performance.

on the same stimulus when it translated across the screen at $7 \%$ s. To optimize MOT performance, gaze must be centered on the stimulus cloud (Fehd and Seiffert, 2008). Therefore, when MOT is done while the cloud translates, pursuit of the cloud would be necessary to optimize performance. Note that no explicit pursuit or fixation target was provided while performing the MOT tasks.

MOT performance (probe identification accuracy) declined for all subjects as the number of targets increased (Fig. 3). This occurred both when the cloud remained stationary and when it translated across the screen. A $2 \times 3$ repeated-measures ANOVA was conducted on the percentage correct probe identification with the stimulus cloud motion (stationary or translating) and the number of tracked targets as variables. The main effect of the number of MOT targets was significant $\left(F_{(2,6)}=46.02, p=\right.$ $0.0002)$, but the main effect of cloud motion was not $\left(F_{(1,3)}=0.1\right.$, $p=0.76)$. The interaction between cloud motion and number of MOT targets was also not significant $\left(F_{(2,6)}=0.05, p=0.95\right)$. Therefore, simultaneous pursuit had no effect on MOT performance, implying that integration of the stimulus to drive pursuit did not usurp attention from the MOT task.

When attentional resources are shared between two simultaneous tasks, it is not always the case that performance on both is compromised; sometimes, only one process suffers (Khurana and Kowler, 1987). Therefore, even though MOT performance was unaffected by pursuit, it cannot be concluded that the two did not share resources without looking at whether pursuit was poorer during the MOT task. To investigate this, we characterized two hallmark measures of smooth pursuit quality, gain and saccade frequency, with and without a simultaneous MOT task (Fig. 4). The analysis was restricted to the steady-state period of pursuit during attentive tracking of the targets (500-2700 ms after motion onset). In Figure $4 a$, it can be seen in one observer that the magnitude of eye velocity was roughly the same with different numbers of dots, with and without the MOT task. Figure $4 b$ shows that pursuit gain does not systematically decline as a result of performing the MOT task, or as the number of targets increase. A two-way repeated-measures ANOVA on the steadystate gain data showed that performing the MOT task did not affect pursuit gain $\left(F_{(1,3)}=2.09, p=0.24\right)$, nor did increasing the number of MOT targets $\left(F_{(2,6)}=2.96, p=0.13\right)$. The interaction between performing the task and number of MOT targets was also not significant $\left(F_{(2,6)}=0.30, p=0.75\right)$. 
We also used saccade frequency as a measure of pursuit quality, as a greater number of saccades is an indicator of poor pursuit, such as when damage or disease affects the pursuit system (White et al., 1983; Fletcher and Sharpe, 1988; Moser et al., 1990). A two-way repeated-measures ANOVA confirmed that performing the MOT task did not have a significant effect on saccade frequency $\left(F_{(1,3)}=0.69, p=\right.$ $0.468)$. Interestingly, increasing the number of targets did not increase saccade frequency, but rather it significantly reduced it $\left(F_{(2,6)}=5.89, p=0.038\right)$. The interaction between task and target number was not significant $\left(F_{(2,6)}=3.38, p=0.10\right.$; see Fig. $4 c$ ). Thus even under the greatest attentional demands of the MOT task, pursuit remained optimal, further evidence that the integration process for pursuit eye movements is independent from the process performing MOT and may be relatively inattentive.

Although the results of the dual-task experiment suggest that integration and segregation occur simultaneously on the same stimulus, it is possible that only the motions of the unattended dots were integrated, allowing segregation to occur on the remaining subset of dots. To test for this possibility, a two-condition control experiment was conducted on three observers who (1) pursued a two-dot stimulus without the MOT task, and (2) pursued a four-dot stimulus while performing MOT on two of the targets. If only the nontarget dots were integrated during pursuit of the four-dot stimulus, then pursuit of the four-dot stimulus should be no different from pursuit of the two-dot stimulus. On the contrary, pursuit was worse for the two-dot stimulus. Two observers showed significantly lower pursuit gain for the two-dot stimulus $\left(\mathrm{EP}: t_{(59)}=10.09, p=1.9 \times 10^{-14}\right.$; ML: $t_{(59)}=$ $\left.16.00, p=4.10 \times 10^{-23}\right)$. Whereas the other observer (SW) did not show a difference in overall gain $\left(t_{(59)}=0.03, p=0.98\right)$, his eye velocity was far more variable (higher SD) for the two-dot stimulus $\left(t_{(59)}=-10.02, p=2.42 \times 10^{-14}\right)$. These results provide evidence that observers were integrating all dot motions to drive pursuit and not relying only on the nontarget dots.

Alternatively, the pursuit system was using a predictive strategy that did not require integration of the local elements. This was possible, at least after the first few trials, because the stimulus cloud always moved at the same constant velocity in our experiment, and pursuit can continue along predictable trajectories when a target disappears (Madelain and Krauzlis, 2003). To test for this, in a control experiment the translational speed of the cloud changed unpredictably in a random subset of trials. There were nine total dot elements in the stimulus, and four MOT targets. The motions of the local elements within the container were the same as in the previous experiments, and as before, the cloud initially translated across the display at $7 \%$ s. However, in $60 \%$ of trials, the translational speed of the cloud either randomly increased to $11.5 \%$ or decreased to $2.3 \%$ at a random time after motion onset $(700-2000 \mathrm{~ms})$. The stimulus traveled at the new speed for $500 \mathrm{~ms}$, then returned to $7 \% \mathrm{~s}$ for the remainder of the trial. Observers pursued the stimulus, and simultaneously performed the MOT task. Each block comprised 72 trials and speed increases and decreases were equally likely. Three new observers (one female and two males), two of whom were naive to the purpose of the experiment, completed two blocks of trials each.

Figure $5 a$ shows representative average eye traces of one observer during pursuit of the cloud as its speed increased, decreased, or remained constant, with and without the task (solid and dashed lines). Her pursuit was sensitive to the speed change, although the change duration was too short to allow eye velocity to reach steady-state. To quantify the response, mean horizontal eye velocity was calculated in the $200 \mathrm{~ms}$ interval preceding the end of the speed change, and compared with baseline eye velocity in the $200 \mathrm{~ms}$ before the change (Fig. 5b). Pursuit reliably reacted to both increases and decreases in stimulus speed. A paired $t$ test comparing eye velocity changes to zero verified this happened when stimulus speed increased $\left(t_{(5)}=4.74, p=0.005\right)$ or decreased $\left(t_{(5)}=-11.17, p=0.0001\right)$. Note one observer (JF) showed a decrease in eye velocity when the stimulus speed increased when not performing the task, possibly because motivation to follow the change was lacking when individual elements did not require attention. Therefore, observers followed the speed changes in the stimulus successfully during MOT, and were not using a predictive strategy to pursue the cloud. Nor was attention being diverted away from the task to detect the speed change, as observers performed MOT as well with the change as without (paired $t$ test: $t_{(5)}=-0.67, p=0.53$; Fig. $5 c$ ). 


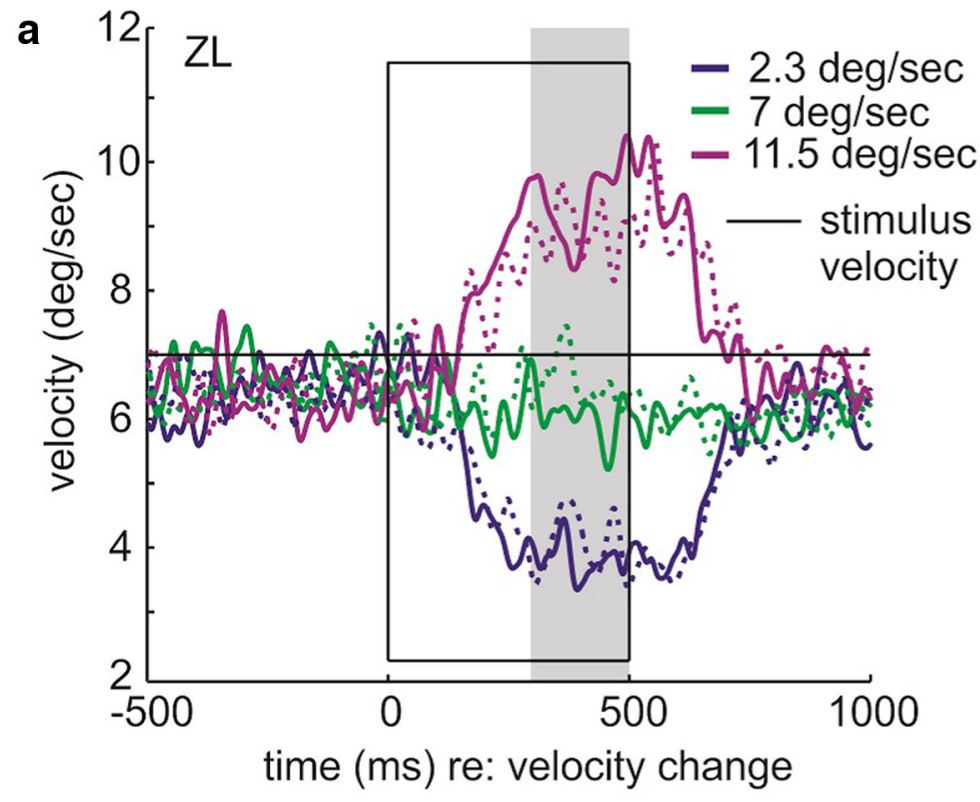

b
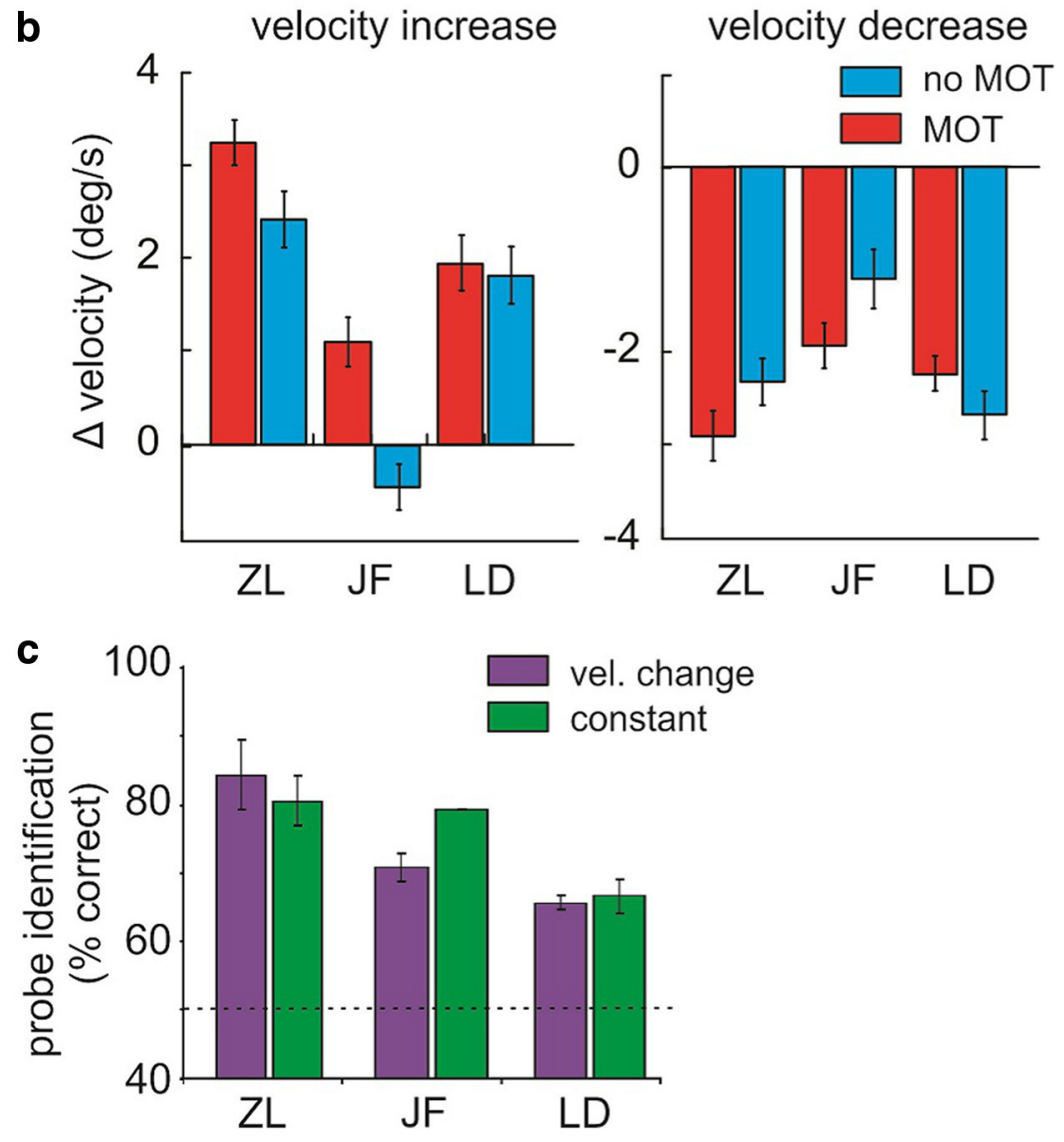

Figure 5. $\quad \boldsymbol{a}$, Representative average eye velocity traces for one observer in response to transient changes in stimulus speed. Solid and dashed lines correspond to MOT task and no task conditions, respectively. Time 0 represents the moment of stimulus speed change and the black lines indicate stimulus speeds. The gray area indicates the analysis region for determining horizontal eye velocity after the change. $\boldsymbol{b}$, Average changes in horizontal eye velocity following the stimulus speed change, while performing MOT and not, for each observer. c, MOT performance with and without the speed change. Error bars indicate SEM. Horizontal dashed lines indicate $50 \%$ (chance) performance.

\section{Discussion}

In the current study, we found that segregation of local motions to support MOT and integration of the same local motions to drive smooth pursuit can occur simultaneously with no penalty to either process. In the experiments, observers pursued a small cloud of randomly moving elements while attentionally tracking a subset of them. Even when the difficulty of the MOT task was high enough to degrade performance, integration remained unimpaired, and pursuit was as good as it was during the "easier" MOT task or without it. Correspondingly, MOT performance during pursuit was no different from performance on a nontranslating stimulus. A control experiment excluded that only the nonattended elements were integrated and pursued to allow segregation of the attended elements. A second control experiment ruled out that the pursuit system was using a predictive strategy to follow the stimulus.

We interpret our results under the "multifocal attention" theory of MOT performance (Cavanagh and Alvarez, 2005), which posits that attention is divided among the different tracked elements during MOT. This theory differs from the early FINST model (Pylyshyn and Storm, 1988), which states that MOT tracking is automatic, requiring no attentional resources. More recent results, however, support the multifocal theory. In a study by Oksama and Hyona (2004), increasing tracking duration and load degraded MOT performance, and visuospatial working memory and attention switching ability were significant predictors of MOT performance. Both results support the idea that MOT uses cognitive processes and is not automatic. In another study, tracking precision increased when a distractor moved close to a target, suggesting that attention is dynamically and adaptively distributed to targets in crowded situations (Iordanescu et al., 2009).

To our knowledge, simultaneous integration and segregation of the same motion elements has not yet been demonstrated. Other work has shown that observers can perform MOT on a moving stimulus (Liu et al., 2005). However, in that study, eye movements were not recorded, and therefore it was not documented whether the quality of pursuit was compromised, or whether the stimulus was pursued at all. Another study indirectly suggested that integration and segregation could happen simultaneously (Watamaniuk and McKee, 1998). In that experiment, observers were asked to judge either the local velocity of a single dot embedded in a moving multidot RDC, or the global velocity of the RDC. To judge the dot's velocity, the dot's motion had to be segregated from the global motion of the RDC. To judge the RDC's velocity, the motion of the individual elements had to be integrated. Even when observers were not in- 
formed which judgment they were to make until after the display stopped moving, they performed the task successfully, indicating either that integration and segregation were occurring simultaneously, or that the motion was stored in memory, and the appropriate operation was selected after the observer was instructed which motion to judge.

However, in the above experiment, the dot did not require integration and the background did not require segregation. The distinct spatial locations of the local and global elements made it possible for the dot and background to stimulate different populations of neurons, thereby allowing one population to integrate and another to segregate. Therefore, it is feasible that both operations could occur simultaneously in the same pathway. In our experiment, because the elements used for integration and segregation were the same and thus not spatially distinct, they would be processed by the same neurons. Because integration is mathematically opposed to segregation, it is unlikely that the same neurons can perform both operations simultaneously. Therefore, our results suggest that different pathways or different populations of neurons subserve integration and segregation.

MOT could be performed by tracking the positions of the moving targets, and not their motions, thereby not using motion segregation. Evidence for this comes from work in which targets were occluded for a short time, and reappeared either at positions predicted by their motions, positions where they were turned off, or previously traversed "rewound" positions (Keane and Pylyshyn, 2006). It was concluded from this study that prediction is not used during MOT because performance was worse when targets reappeared in their predicted positions. However, decreased performance does not indicate that observers were not using motion prediction at all, because motion information was available in all conditions. A performance decrement might have been observed simply because predicted positions are less certain than previously traversed ones.

A later study addressed these problems directly (Fencsik et al., 2007). These authors first replicated the results of Keane and Pylyshyn (2006), then added a condition in which the targets stopped moving before the occlusion, effectively eliminating motion cues in the task. This manipulation degraded performance, providing evidence that motion is used to perform MOT. Other recent work provides additional evidence that motion is used in MOT. Observers predict the location to which tracked targets' motion would take them (Iordanescu et al., 2009), and superimposing conflicting motion on moving targets disrupts attentional tracking (St Clair et al., 2010). Furthermore, motion circuitry in the middle temporal area (MT) is activated during MOT (Howe et al., 2009).

In our experiment, we used a dual-task paradigm in which observers simultaneously performed a perceptual task, MOT, and a motor task, ocular pursuit. Dual-task paradigms are typically used to determine whether attentional resources are shared between two neural systems (Navon and Gopher, 1979). The underlying assumption of these paradigms is that attentional resources are finite. Therefore, if two systems share attention, activating them simultaneously will usurp resources from one or both of the systems, and compromise performance on one or both of the tasks. We first measured MOT performance for increasing levels of task difficulty, and found a systematic decrease in performance for all observers. When we added the pursuit task, MOT performance at all difficulty levels was unaffected, and pursuit performance remained unchanged from the conditions in which no MOT task was performed. When these results are interpreted in the standard dual-task framework, the conclusion is that attentional resources for MOT are not shared with those used to pursue the multidot stimulus, and thus the opposing processes of segregation and integration used to drive these tasks are independent. Our explanation is consistent with this, but more specific. We think that pursuit of the multidot stimulus does not share attention with MOT because pursuit of larger stimuli that extend beyond the fovea and the integration process driving it are relatively inattentive.

This theory is consistent with other work suggesting that the system used to pursue larger, natural objects is reflexive-like, leaving attention resources free to inspect object features (Heinen et al., 2011; Jin et al., 2013). In those studies, observers performed a secondary attention task during pursuit of a small spot stimulus with or without a large, moving RDC background. In one experiment, observers had to identify which of five dots briefly dimmed (Heinen et al., 2011). Performance on the dimming task was better when the RDC was present; indicating that pursuit of a large stimulus was relatively inattentive. In another experiment, observers made saccades during pursuit to targets superimposed on the RDC, or moving with a single spot (Jin et al., 2013). Shorter saccade latency was found during pursuit with the RDC, evidence that the saccadic system shared attention resources with the system that pursued the spot, as well as additional evidence for a less attentive system generating pursuit of the RDC. Note that in these experiments, the task elements were different from those composing the RDC, and therefore did not rule out that integration and segregation were being performed by different sets of motion detectors in the same pathway.

Which structures might compose the pathways underlying simultaneous MOT and pursuit of large stimuli? Both functions likely involve the motion-processing regions in MT and middle superior temporal cortex, as pursuit (Komatsu and Wurtz, 1988) and MOT-related activation (Culham et al., 1998) has been observed in these structures. For MOT, regions in parietal and frontal cortex that are involved in MOT would likely be recruited (Culham et al., 1998), as well as V4 (Mitchell et al., 2007). For pursuit, we believe there exists a relatively reflexive and subconscious neural mechanism devoted to pursuing objects that extend beyond the fovea and stimulate peripheral retina. This pathway recruits circuitry within the primitive system that generates the optokinetic reflex (OKR). As a subsystem of ocular following, OKR circuitry may have been modified through evolution to pursue objects that are common in natural scenes, such as proximal people or animals. Supporting this notion, the nucleus of the optic track, which is commonly thought to drive OKR (Hoffmann et al., 1988; Kato et al., 1988; Schiff et al., 1988), contains single neurons that respond during smooth pursuit and OKR (Mustari and Fuchs, 1990; Ilg and Hoffmann, 1996) and might be involved in pursuit of larger objects. This modern, yet still mostly automatic, OKR circuitry releases attention for the inspection of an object's features, which uses a foveate system of fixation and saccades.

\section{References}

Acker W, Toone B (1978) Attention, eye tracking and schizophrenia. Br J Soc Clin Psychol 17:173-181. CrossRef Medline

Alvarez GA, Franconeri SL (2007) How many objects can you attentively track? Evidence for a resource-limited tracking mechanism. J Vis 7(13):14 1-10. CrossRef Medline

Brainard DH (1997) The psychophysics toolbox. Spat Vis 10:433-436. CrossRef Medline

Brezinová V, Kendell RE (1977) Smooth pursuit eye movements of schizophrenics and normal people under stress. Br J Psychiatry 130:59-63. CrossRef Medline 
Cavanagh P, Alvarez GA (2005) Tracking multiple targets with multifocal attention. Trends Cogn Sci 9:349-354. CrossRef Medline

Culham JC, Brandt SA, Cavanagh P, Kanwisher NG, Dale AM, Tootell RB (1998) Cortical fMRI activation produced by attentive tracking of moving targets. J Neurophysiol 80:2657-2670. Medline

Fehd HM, Seiffert AE (2008) Eye movements during multiple object tracking: where do participants look? Cognition 108:201-209. CrossRef Medline

Fencsik DE, Klieger SB, Horowitz TS (2007) The role of location and motion information in the tracking and recovery of moving objects. Percept Psychophys 69:567-577. CrossRef Medline

Fletcher WA, Sharpe JA (1988) Smooth pursuit dysfunction in Alzheimer's disease. Neurology 38:272-277. CrossRef Medline

Heinen SJ, Watamaniuk SN (1998) Spatial integration in human smooth pursuit. Vision Res 38:3785-3794. CrossRef Medline

Heinen SJ, Jin Z, Reeves A, Watamaniuk SNJ (2011) Flexibility of foveal attention during ocular pursuit. J Vis 11(2):9 1-12. CrossRef Medline

Hoffmann KP, Distler C, Erickson RG, Mader W (1988) Physiological and anatomical identification of the nucleus of the optic tract and dorsal terminal nucleus of the accessory optic tract in monkeys. Exp Brain Res 69:635-644. Medline

Howe PD, Pinto Y, Horowitz TS (2010) The coordinate systems used in visual tracking. Vis Res 50:2375-2380. CrossRef Medline

Howe PD, Horowitz TS, Morocz IA, Wolfe J, Livingstone MS (2009) Using fMRI to distinguish components of the multiple object tracking task. J Vis 9(4):10 1-11. CrossRef Medline

Ilg UJ, Hoffmann KP (1996) Responses of neurons of the optic tract and the dorsal terminal nucleus of the accessory optic tract in the awake monkey. Eur J Neurosci 8:92-105. CrossRef Medline

Iordanescu L, Grabowecky M, Suzuki S (2009) Demand-based dynamic distribution of attention and monitoring of velocities during multiple-object tracking. J Vis 9(4):1 1-12. CrossRef Medline

Jin Z, Reeves A, Watamaniuk SN, Heinen SJ (2013) Shared attention for smooth pursuit and saccades. J Vis 13(4):7 1-12. CrossRef Medline

Kato I, Harada K, Hasekawa T, Ikarashi T (1988) Role of the nucleus of the optic tract in monkeys in optokinetic nystagmus and optokinetic after nystagmus. Brain Res 474:16-26. CrossRef Medline

Keane BP, Pylyshyn ZW (2006) Is motion extrapolation employed in multiple object tracking? Tracking as a low-level, nonpredictive function. Cogn Psychol 52:346-368. CrossRef Medline

Kerzel D, Souto D, Ziegler NE (2008) Effects of attention shifts to stationary objects during steady-state smooth pursuit eye movements. Vis Res 48: 958-969. CrossRef Medline

Khurana B, Kowler E (1987) Shared attentional control of smooth eye movement and perception. Vis Res 27:1603-1618. CrossRef Medline

Komatsu H, Wurtz RH (1988) Relation of cortical areas MT and MST to pursuit eye movements: III. Interaction with full field visual stimulation. J Neurophysiol 60:621-644. Medline

Liu G, Austen EL, Booth KS, Fisher BD, Argue R, Rempel MI, Enns JT (2005) Multiple-object tracking is based on scene, not retinal, coordinates. J Exp Psychol Hum Percept Perform 31:235-247. CrossRef Medline
Madelain L, Krauzlis RJ (2003) Effects of learning on smooth pursuit during transient disappearance of a visual target. J Neurophysiol 90:972-982. CrossRef Medline

Mitchell JF, Sundberg KA, Reynolds JH (2007) Differential attentiondependent response modulation across cell classes in macaque visual area V4. Neuron 55:131-141. CrossRef Medline

Moser A, Kompf V, Arolt V, Resch T (1990) Quantitative analysis of eye movements in schizophrenia. Neuro-Ophthalmology 10:73-80. CrossRef

Mustari MJ, Fuchs AF (1990) Discharge patterns of neurons in the pretectal nucleus of the optic tract (NOT) in the behaving primate. J Neurophysiol 64:77-90. Medline

Navon D, Gopher D (1979) On the economy of the human-processing system. Psychol Rev 86:214-255. CrossRef

Oksama L, Hyona J (2004) Is multiple object tracking carried out automatically by an early vision mechanism independent of higher order cognition? An individual difference approach. Vis Cogn 11:631-671. CrossRef

Pelli DG (1997) The VideoToolbox software for visual psychophysics: transforming numbers into movies. Spat Vis 10:437-442. CrossRef Medline

Pylyshyn ZW, Annan V Jr (2006) Dynamics of target selection in multiple object tracking (MOT). Spat Vis 19:485-504. CrossRef Medline

Pylyshyn ZW, Storm RW (1988) Tracking multiple independent targets: evidence for a parallel tracking mechanism. Spat Vis 3:179-197. CrossRef Medline

Schiff D, Cohen B, Raphan T (1988) Nystagmus induced by stimulation of the nucleus of the optic tract in the monkey. Exp Brain Res 70:1-14. Medline

Schütz AC, Spering M, Braun DI, Gegenfurtner KR (2009) Smooth pursuit eye movements and the segregation of coherent motion [abstract]. J Vis 9(8):426. CrossRef

St Clair R, Huff M, Seiffert AE (2010) Conflicting motion information impairs multiple object tracking. J Vis 10(4):18 1-13. CrossRef Medline

Steinbach MJ (1976) Pursuing the perceptual rather than the retinal stimulus. Vis Res 16:1371-1376. CrossRef Medline

Stone LS, Beutter BR, Lorenceau J (2000) Visual motion integration for perception and pursuit. Perception 29:771-787. CrossRef Medline

Watamaniuk SN, Duchon A (1992) The human visual system averages speed information. Vis Res 32:931-941. CrossRef Medline

Watamaniuk SN, Heinen SJ (1999) Human smooth pursuit direction discrimination. Vis Res 39:59-70. CrossRef Medline

Watamaniuk SN, McKee SP (1998) Simultaneous encoding of direction at a local and global scale. Percept Psychophys 60:191-200. CrossRef Medline

White OB, Saint-Cyr JA, Tomlinson RD, Sharpe JA (1983) Oculomotor deficits in Parkinson's disease II: control of the saccadic and smooth pursuit systems. Brain 106:571-587. CrossRef Medline

Williams DW, Sekuler R (1984) Coherent global motion percepts from stochastic local motions. Vis Res 24:55-62. CrossRef Medline

Yantis S (1992) Multielement visual tracking: attention and perceptual organization. Cogn Psychol 24:295-340. CrossRef Medline 